\title{
The interaction between supermassive black holes and globular clusters
}

\author{
Mario Spera ${ }^{1,2}$, Manuel Arca-Sedda ${ }^{2,3}$ \\ and Roberto Capuzzo-Dolcetta ${ }^{2}$ \\ ${ }^{1}$ INAF-Osservatorio Astronomico di Padova, Vicolo dell'Osservatorio 5, I-35122, Padova, Italy \\ email: mario.spera@oapd.inaf.it \\ ${ }^{2}$ Sapienza-Universitá di Roma, P.le A. Moro 5, I-00165 Rome, Italy \\ ${ }^{3}$ Universitá di Tor Vergata, Via O. Raimondo 18, I-00173 Rome, Italy
}

\begin{abstract}
Almost all galaxies along the Hubble sequence host a compact massive object (CMO) in their center. The CMO can be either a supermassive black hole (SMBH) or a very dense stellar cluster, also known as nuclear star cluster (NSC). Generally, heavier galaxies (mass $\gtrsim 10^{11} \mathrm{M}_{\odot}$ ) host a central SMBH while lighter show a central NSC. Intermediate mass hosts, instead, contain both a NSC and a SMBH. One possible formation mechanisms of a NSC relies on the dry-merger (migratory) scenario, in which globular clusters (GCs) decay toward the center of the host galaxy and merge. In this framework, the absence of NSCs in high-mass galaxies can be imputed to destruction of the infalling GCs by the intense tidal field of the central SMBH. In this work, we report preliminary results of $N$-body simulations performed using our high-resolution, direct, code HiGPUs, to investigate the effects of a central SMBH on a single GC orbiting around it. By varying either the mass of the SMBH and the mass of the host galaxy, we derived an upper limit to the mass of the central SMBH, and thus to the mass of the host, above which the formation of a NSC is suppressed.
\end{abstract}

Keywords. galaxies: star clusters, methods: n-body simulations

\section{Introduction}

The innermost regions of galaxies often host a very compact stellar cluster with a typical half-light radius, $r_{\mathrm{hl}}$, of few parsecs, luminosity $\sim 10^{7} \mathrm{~L}_{\odot}$ and total mass $\sim 10^{7} \mathrm{M}_{\odot}$. These compact structures are known as nuclear star clusters (NSCs) (Böker et al. 2004; Côté et al. 2006; Turner et al. 2012). NSCs are observed in galaxies of all the Hubble types and, sometimes, they co-exist with a central supermassive black hole (SMBH) (Graham \& Spitler 2009). For instance, the Milky Way hosts both a NSC with mass $\sim 10^{7} \mathrm{M}_{\odot}$ and a SMBH of mass $\sim 4 \times 10^{6} \mathrm{M}_{\odot}$ (Schödel et al. 2009).

Two (not exclusive) mechanisms have been proposed for NSC formation:

(a) dissipative: in this framework the NSC should form thanks to a continuous radial inflow of gas and a subsequent, in situ, star formation (Milosavljević 2004; Bekki 2007);

(b) dissipationless: this is also known as dry-merging scenario in which globular clusters (GCs) decay towards the galactic center via dynamical friction forming and accreting the NSC (Tremaine et al. 1975; Capuzzo-Dolcetta 1993; Capuzzo-Dolcetta \& Miocchi 2008).

While the in-situ scenario is purely speculative, the dry-merging scenario has been quantitatively investigated and matches observational data and correlations (Arca-Sedda \& Capuzzo-Dolcetta 2014; Antonini et al. 2012). Anyway, likely the actual formation mechanism can be the result of some combination between the dissipative and the dissipationless processes. Moreover, the presence of a central SMBH could significantly alter the process of formation and growth of the NSC; many authors have shown that the 
masses of the SMBH, of the galaxy and of the NSC are strongly related. In particular, there is observational evidence of a clear distinction between low-mass galaxies (with mass $\lesssim 10^{10} \mathrm{M}_{\odot}$ ), whose nuclei are dominated by a NSC, and heavier galaxies, dominated by a SMBH (Scott \& Graham 2013).

In this work, and in the framework of the dry-merging scenario, we investigate the interaction between SMBHs and GCs, in order to determine if the mutual influence may play a role in the co-existence of SMBHs and NSCs. We give here preliminary results of a more extended work still in progress (Arca-Sedda et al. 2015).

We approach the problem by means of high-precision, direct, $N$-body simulations following the dynamical evolution of several astrophysical systems composed of a galaxy bulge, a central SMBH and a GC moving on different orbits. To this purpose, we used the $N$-body code HiGPUs (Capuzzo-Dolcetta et al. 2013) which, thanks to the hardware acceleration given by graphics processing units, allowed us to use $\sim 1 \mathrm{M}$ particles in our simulations, obtaining scientific results with high spatial resolution.

\section{Model}

Globular cluster The test GC in our simulations is built according to a King's mass density profile with central potential parameter $W_{0}=6$, a King's radius $r_{\mathrm{k}}=0.24 \mathrm{pc}$ and a total mass $M_{\mathrm{GC}}=10^{6} \mathrm{M}_{\odot}$.

In all the investigated cases, the GC is initially placed at $50 \mathrm{pc}$ from the central $\mathrm{SMBH}$. Therefore, we assume that the GC has already decayed toward the inner galactic region, where the presence of the $\mathrm{SMBH}$ may play an important dynamical role. It is worth noting that the choice of a large initial GC mass $\left(10^{6} \mathrm{M}_{\odot}\right)$ is motivated by the requirement of orbital shrinking via dynamical friction in less than a Hubble time.

Galaxies and central black holes Since direct $N$-body simulations cannot handle more than $\sim 10^{6}$ particles in an efficient way, we decided to model our galaxies by sampling only their central regions. In this work, we focus our attention on elliptical galaxies represented as Dehnen's mass density profiles family (at varying $\gamma$, see Dehnen 1993), $\rho_{D}(r)$, truncated (McMillan \& Dehnen 2007) as

$$
\rho_{t r}(r)=\rho_{D}(r) \operatorname{sech}\left(\frac{r}{r_{\text {cut }}}\right) .
$$

The $\rho_{t r}(r)$ profile falls off as $e^{-r / r_{\text {cut }}}$ for $r \gtrsim r_{\text {cut }}$ allowing a good representation of the region of interest with a reasonable number of particles. The mass value of the central SMBH is assigned according to the formula by Scott \& Graham (2013)

$$
\log \left(\frac{M_{\mathrm{BH}}}{\mathrm{M}_{\odot}}\right)=1.37 \log \left(\frac{M_{\mathrm{gal}}}{10^{11} \mathrm{M}_{\odot}}\right)+8.06 .
$$

In this work we spanned the mass ranges $10^{10} \mathrm{M}_{\odot}<M_{\text {gal }}<3.2 \times 10^{11} \mathrm{M}_{\odot}$ and $5 \times$ $10^{6} \mathrm{M}_{\odot}<M_{\mathrm{BH}}<5 \times 10^{8} \mathrm{M}_{\odot}$. Table 1 summarizes the parameters adopted in each simulation.

\section{Results for GC circular orbits}

For each galaxy model, we made three simulations corresponding to a circular, an eccentric $(e \sim 0.75)$ and a radial orbit of the GC. In this preliminary work we report the results of the GC moving on a circular orbit. 
Table 1. Parameters of the models. $M_{\mathrm{gal}}$ and $M_{\mathrm{gal}, \mathrm{cut}}$ are the total and truncated (in dependence on $r_{\text {cut }}$ ) galaxy masses, $M_{\mathrm{BH}}$ is the SMBH mass; $r_{s}$ and $\gamma$ is the parameter of the galactic Dehnen's profile; $N_{\mathrm{gal}}$ and $N_{\mathrm{GC}}$ are the numbers of particles used in our simulations for the galaxy and for the GC.

\begin{tabular}{cccccccc}
\hline $\begin{array}{c}\boldsymbol{M}_{\text {gal }} \\
\left(\mathrm{M}_{\odot}\right)\end{array}$ & $\begin{array}{c}\boldsymbol{M}_{\mathrm{BH}} \\
\left(\mathrm{M}_{\odot}\right)\end{array}$ & $\begin{array}{c}\boldsymbol{r}_{\boldsymbol{s}} \\
(\mathrm{kpc})\end{array}$ & $\begin{array}{c}\boldsymbol{r}_{\text {cut }} \\
(\mathrm{pc})\end{array}$ & $\boldsymbol{\gamma}$ & $\begin{array}{c}\boldsymbol{M}_{\text {gal,cut }} \\
\left(\mathrm{M}_{\odot}\right)\end{array}$ & $\boldsymbol{N}_{\text {gal }}$ & $\boldsymbol{N}_{\mathrm{GC}}$ \\
\hline $10^{10}$ & $5 \times 10^{6}$ & 0.995 & 70 & 0.3 & $3.4 \times 10^{7}$ & $1,018,742$ & 29,832 \\
\hline $3.2 \times 10^{10}$ & $2 \times 10^{7}$ & 1.512 & 70 & 0.3 & $4.1 \times 10^{7}$ & $1,024,025$ & 24,550 \\
\hline $10^{11}$ & $10^{8}$ & 1.917 & 70 & 0.2 & $5.9 \times 10^{7}$ & $1,031,338$ & 17,237 \\
\hline $3.2 \times 10^{11}$ & $5 \times 10^{8}$ & 2.876 & 70 & 0.2 & $6.8 \times 10^{7}$ & $1,033,332$ & 15,243 \\
\hline
\end{tabular}

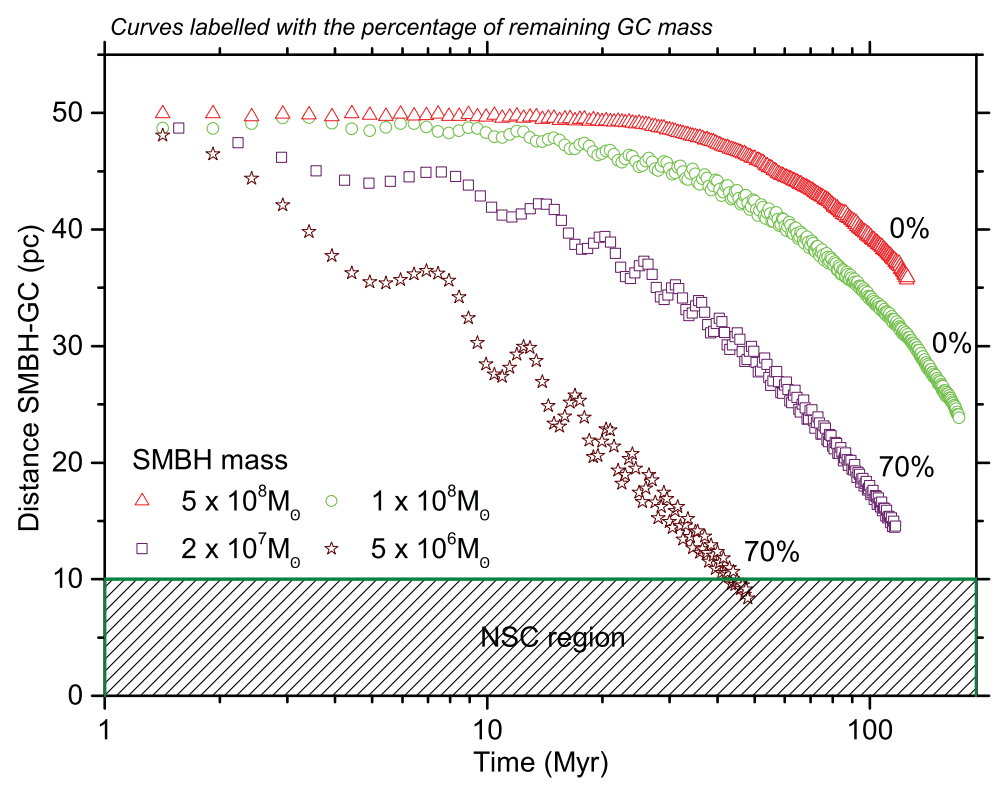

Figure 1. Time evolution of the distance of the decaying GC from the SMBH in the models of Table 1. Each curve is labeled with the percentage to the initial mass that the GC keep bound at the end of each simulation. The shaded area (distances SMBH-GC smaller than $10 \mathrm{pc}$ ) marks the region where a NSC may form and grow.

The four different curves in Fig. 1 refer to the time evolution of the GC galactocentric distance in our four models of galaxy. Fig. 1 shows that heavier SMBHs are able to disrupt the GC before it gets to the NSC region, while the incoming GC can survive to the interaction with the central SMBHs if this is relatively light. In fact, in the case of $M_{\mathrm{BH}}=5 \times 10^{6} \mathrm{M}_{\odot}$, the $\mathrm{GC}$ can come closer than $10 \mathrm{pc}$ to the $\mathrm{SMBH}$ preserving $70 \%$ of its initial mass.

This implies that, in this situation, GCs may indeed significantly contribute to the formation and growth of a NSC while heavier SMBHs tends to protect the galaxy center, preventing a local mass accumulation and favouring the tidal dissolution of incoming GCs. Figure 1 provides also evidence of a transition regime between dynamical friction dominated galaxies (formation and growth of NSC by mergers) and tidal disruption dominated galaxies (no NSC). Our, preliminary, simulations suggest the transition lies in the range of SMBHs masses between $2 \times 10^{7} \mathrm{M}_{\odot}$ and $10^{8} \mathrm{M}_{\odot}$. 


\section{Conclusions}

We presented preliminary results about the problem of the dearth of NSCs in high mass (elliptical) galaxies. We performed some high-precision, direct $N$-body simulations to investigate the dynamical fate of a massive $\mathrm{GC}$ orbiting the inner region of a galaxy. We showed that a SMBH heavier than $\sim 10^{8} \mathrm{M}_{\odot}$ can efficiently disrupt the infalling GC before it gets to what we called NSC region, and therefore it may inhibit the formation process, by subsequent merging events, of a NSC. On the other hand, the incoming GC survives the interaction with lighter BHs and, thus, can contribute to the formation and growth of a NSC. To conclude, our simulation results are a reliable confirmation of the important role played by a massive central black hole on the infalling GCs, as first pointed out by Capuzzo-Dolcetta (1993) and, more recently, by Antonini (2013). Nevertheless, a firmer statement about the topic studied here deserves:

(a) a wider range of initial conditions for both the GC structure and its initial orbital parameters;

(b) an extension of the galaxy models, to determine more precisely the threshold in $M_{\mathrm{BH}}$ below which the dry-merging of GCs easily allows the formation of a NSC;

(c) a better $N$-body sampling of our models, possible by taking advantage of the next generation hardware and software.

\section{Acknowledgements}

MS thanks M. Mapelli for useful discussions, and acknowledges financial support from the MIUR through grant FIRB 2012 RBFR12PM1F. MAS acknowledges financial support provided by MIUR through the grant PRIN 2010 LY5N2T 005.

\section{References}

Antonini, F. 2013, ApJ, 763, 62

Antonini, F., Capuzzo-Dolcetta, R., Mastrobuono-Battisti, A., \& Merritt, D. 2012, ApJ, 750, 111

Arca-Sedda, M. \& Capuzzo-Dolcetta, R. 2014, MNRAS, 444, 3738-3755

Arca-Sedda, M., Capuzzo-Dolcetta, R., \& Spera, M. 2015, submitted to MNRAS (arXiv:1510.01137)

Bekki, K. 2007, PASA, 24, 77

Böker, T., Sarzi, M., McLaughlin, D. E., et al. 2004, AJ, 127, 105

Capuzzo-Dolcetta, R. 1993, ApJ, 415, 616

Capuzzo-Dolcetta, R. \& Miocchi, P. 2008, ApJ, 681, 1136

Capuzzo-Dolcetta, R., Spera, M., \& Punzo, D. 2013, JCP, 236, 580

Côté, P., Piatek, S., Ferrarese, L., et al. 2006, ApJS, 165, 57-94

Dehnen, W. 1993, MNRAS, 265, 250

Graham, A. W. \& Spitler, L. R. 2009, MNRAS, 397, 2148

McMillan, P. J. \& Dehnen, W. 2007, MNRAS, 378, 541

Milosavljević, M. 2004, ApJ, 605, L13

Schödel, R., Merritt, D., \& Eckart, A. 2009, A\&A, 502, 91

Scott, N. \& Graham, A. W. 2013, ApJ, 763, 76

Tremaine, S. D., Ostriker, J. P., \& Spitzer, L., Jr. 1975, ApJ, 196, 407

Turner, M. L., Côté, P., Ferrarese, L., et al. 2012, ApJS, 203, 5 